\title{
The path of embryo selection for improving IVF
}

\section{success rates}

\section{Opinion}

Reproductive endocrinologists and clinical embryologists have the difficult and important task of selecting which of the available embryos created after in vitro fertilization (IVF) to transfer-those they think that are most likely to provide the best outcome for their patients. If they fail to identify and predict the embryos which are more likely to implant the result will be multiple-gestation pregnancies or negative IVF results. ${ }^{1,2}$ The main goal of IVF is to precisely identify the most appropriate embryo(s) for transfer., ${ }^{3,4}$ Although embryo grading has been proposed as the most appropriate system for selecting the right embryo(s), the fact that it is based on the assessment of morphological characteristics in an easy and non-invasive way make it subjective and with a limited predictive value which often results in different interpretations of embryo quality., $2,5,6$

The efficacy of blastocyst culture revealed a new fast and secure method to identify competent embryos. A self-selection system in which embryo viability to blastocyst stage can maximize the chance of implantation. ${ }^{7,8}$ Although the philosophy of blastocyst culture is to improve both uterine and embryonic synchronicity and result in higher implantation rates, ${ }^{9,10}$ it involves prolonged culture, which currently results in blastocyst development in approximately half of all good-quality day 3 (D3) embryos. ${ }^{11,12}$ Therefore only if the patient has many embryos will benefit from embryo transfer at the blastocyst stage than with cleavage-stage embryo transfer. ${ }^{13}$ Furthermore, some evidence (even though conflicting) suggests that prolonged embryo culture may also increase the risk of preterm delivery, low birth weight, epigenetic disorders, monozygotic twinning and other longterm health issues. ${ }^{11,12,14,15}$ Hence, reliable prediction of blastocyst formation as early as on day 3 may be useful, especially for IVF patients with repeated failures after D3 embryo-transfers. Despite the duration of embryo culture, morphology assessment is still used to select the best-quality embryos when several embryos with good similar morphological characteristics are available for transfer or cryopreservation.

The identification of embryos that are most likely to result in a pregnancy can be improved by two different paths: by predicting the embryos that are more likely to develop correctly and finally implant or by non-selecting the embryos that have less possibilities to develop further and therefore may have lower implantation potential. ${ }^{16}$ Therefore a further improvement in the design of innovative approaches can improve embryo selection at the time of transfer, thereby maximizing the chances of implantation and the delivery of a healthy baby.

Because of its noninvasiveness to the embryo and its rapid adoption in the IVF units, time-lapse microscopy is particularly well suited not only to identify new selection criteria but also provide information about the dynamic changes during the pre implantation period $^{17,18}$ Time-lapse imaging provides a non invasive technique of predictive parameters based on developmental kinetics by detecting the dynamics of early embryo development. The main challenge of applying time-lapse microscopy in everyday IVF practice is ensuring

\author{
Volume 3 Issue 2 - 2016
}

\author{
Makrakis E, Dinopoulou V, Giannaris D \\ Embryo ART Unit, Greece \\ Correspondence: Makrakis E, Embryo A.R.T. Unit, 194, \\ Alexandras Avenue, Athens I 1522, Greece, \\ Emailevmakrakis@gmail.com
}

Received: May 24, 2016 | Published: June 03, 2016

embryo culture and light exposure safety, defining and validating predictive parameters using evidence-based data and processing abundant imaging data in real-time. "A picture tells 1000 words", freeing the IVF lab from time restrictions, time-lapse microscopy allows the identification of parameters that may noninvasively predict the developmental potential of a cleavage-stage embryo. ${ }^{17,19}$

Published data suggest that morphokinetic observations can yield valuable information to aid the selection of embryos for transfer ${ }^{15,20}$ In many studies, cell cycle timing parameters that were retrospectively chosen from a variety of images of time-lapse videos have constantly shown strong parallel of human embryo development and implantation potential. ${ }^{19-21}$ Although, even today, there is none prospective, multicenter clinical study, comparing the morphokinetic parameters with traditional embryo selection criteria based on embryo morphological features. Moreover, the embryologist cannot yet take advantage of the time-lapse parameters to ensure, as soon as possible, which embryos have the greatest potential to develop into blastocysts in order to be transferred or cryopreserved. A summary of the papers published in time-lapse research showing that standardization in embryo selection method is necessary.

Lately, it was reported a time-lapse microscopy-enabled embryo assessment test that was developed based on seminal scientific findings about the timings of early embryo development and stateof-the-art software that automatically measures cell division timings and provides quantitative information regarding embryo development potential. ${ }^{15,21}$ The combination of abnormal developmental timings already described and atypical morphokinetic features of embryos ${ }^{21,22}$ could improve further the process of embryo selection by permitting to the embryologist to distinguish the embryos that have good morphology but lower development potential. ${ }^{16}$

Clearly, the attributes of "optimal embryos" based exclusively on embryo morphological criteria given the constant introduction on new technical advances in ART are not enough. As a result, the limitations of morphological and morphokinetic approaches justified the development of new non-invasive "omics" approaches. The development of the "omics" technologies (epigenomics, genomics, 
transcriptomics, proteomics and metabolomics) have as a main aim to identify new non-invasive biomarkers, by providing with a huge amount of information regarding the biological processes involved in reproductive success. Therefore, the "omics" technologies are suitable diagnostic tools to explore differences among embryos with similar morphological features. ${ }^{23}$ Since a single embryo transfer is getting momentum across the IVF clinics, such platforms which diagnose the embryo viability based on the expression of biomarkers will be inevitable to select the embryos for transfer. ${ }^{24}$

Voluminous data suggest that soluble ligands and their receptors mediate human pre implantation embryo development and implantation and could therefore be suitable as noninvasive biomarkers of embryo implantation. ${ }^{25}$ Cortezzi et al. ${ }^{26}$ used a smaller variant of high-performance liquid chromatography combined with electron spray ionization mass spectroscopy to analyze the secretome of day 3 embryo spent media, revealing 25 novel secretory proteins. ${ }^{26}$ In another study by Dominguez et al. ${ }^{27}$ protein arrays, based on antigen-antibody reactions and multiplex technology, have also been used, identifying CXC chemokine ligand (CXCL) 13, stem cell factor (SCF), and tumor necrosis factor receptor (TNFR) 1 in implanted vs. non-implanted embryos. ${ }_{27}$ Butler et al. ${ }^{28}$ reported that, the dominance of hCG (human chorionic gonadotropin) and hCGh (hyperglycosylated hCG) expression seen after blastocyst hatching may be indicative of potential implantation success ${ }^{28}$ and Mains et al., ${ }^{29}$ proved that Apolipoprotein A1 is produced by human preimplantation embryos, and correlated the increased levels in spent culture media with higher morphological grade blastocysts. ${ }^{29}$ Recently, Dominguez et al., ${ }^{30}$ analyzed seven proteins in the embryo spent media (SCF, TNFR1, PIGF-1, IFN-a2, IL-6, CXCL13, and GMCSF) with the use of a beadbased multiplexing technology and combined this data with the exact timing (in hours) of cell cycle duration, blastomere synchrony, and 5-blastomere cleavage with the use of an incubator equipped with time-lapse videography ${ }^{30}$ Finally, only the presence or absence of IL-6 approved to be useful. However, no single biomarker has yet been used in standard clinical practice, mainly due to the differences in culture conditions the complication of the laboratory techniques and the effect of biological variations. ${ }^{31}$

Animal models, so far, have been particularly useful to help scientists analyze whole proteome or single protein markers in follicular fluid and gametes. Several studies have generated large amounts of data, nonetheless, the perfect profile to predict the best oocytes and embryos suitable for implantation are still to be uncovered. ${ }^{32}$ An overview of metabolomics and its complementary role with transcriptomics and proteomics, it highlights how noninvasive foot printing analysis in the embryo spent culture media has many applications for the assessment of the biochemical status of the embryo. ${ }^{33}$

In conclusion, microfluidic devices for embryo culture and analysis may yet prove to be a practical means for the integration and application of these new technologies. Additionally, we are very close to understanding what relationships exist between embryo morphokinetics, gene expression and physiology. In the future, following time-lapse monitoring and analysis of spent culture media for biomarkers will help to formulate comprehensive and robust algorithms for generating a superior embryo selection diagnostic tool. Even if perfection is our dream, improvement is still a reasonable hope and such algorithms are a realistic proposal for improvement in IVF.

\section{Acknowledgements}

None.

\section{Conflicts of interest}

The author declares there is no conflict of interest.

\section{References}

1. Elective single-embryo transfer. Fertil Steril. 2012;97(4):835-842.

2. Paternot G, Wetzels AM, Thonon F, et al. Intra- and interobserver analysis in the morphological assessment of early stage embryos during an IVF procedure:a multicentre study. Reprod Biol Endocrinol. 2011;9:127.

3. Machtinger R, Racowsky C. Morphological systems of human embryo assessment and clinical evidence. Reprod Biomed Online. 2013;26(3):210-21

4. Montag M, Toth B, Strowitzki T. New approaches to embryo selection Reprod Biomed Online. 2013;27(5):539-546.

5. Baxter Bendus AE, Mayer JF, Shipley SK, et al. Interobserver and intraobserver variation in day 3 embryo grading. Fertil Steril. 2006;86(6):1608-1615.

6. Guerif F, Lemseffer M, Leger J, et al. Does early morphology provide additional selection power to blastocyst selection for transfer? Reprod Biomed Online. 2010;21(4):510-519.

7. Glujovsky D, Blake D, Farquhar C, et al. Cleavage stage versus blastocyst stage embryo transfer in assisted reproductive technology. $\mathrm{Co}$ chrane Database Syst Rev. 2012;7:CD002118.

8. Gardner DK, Schoolcraft WB, Wagley L, et al. A prospective randomized trial of blastocyst culture and transfer in in-vitro fertilization. Hum Reprod. 1998;13(12):3434-3440.

9. Papanikolaou EG, D'Haeseleer E, Verheyen G, et al. Live birth rate is significantly higher after blastocyst transfer than after cleavagestage embryo transfer when at least four embryos are available on day 3 of embryo culture. A randomized prospective study. Hum Reprod. 2005;20(11):3198-3203.

10. Gelbaya TA, Tsoumpou I, Nardo LG. The likelihood of live birth and multiple birth after single versus double embryo transfer at the cleavage stage:a systematic review and meta-analysis. Fertil Steril. 2010;94(3):936-945.

11. Niemitz EL, Feinberg AP. Epigenetics and assisted reproductive technology:a call for investigation. Am J Hum Genet. 2004;74(4):599-609.

12. Manipalviratn S, DeCherney A, Segars J. Imprinting disorders and assisted reproductive technology. Fertil Steril. 2009; 91(2):305-315.

13. Diamond MP, Willman S, Chenette P, et al. The clinical need for a method of identification of embryos destined to become a blastocyst in assisted reproductive technology cycles. J Assist Reprod Genet. 2012;29(5):391-396.

14. Kalra SK, Ratcliffe SJ, Barnhart KT, et al. Extended embryo culture and an increased risk of preterm delivery. Obstet Gynecol. 2012;120(1):6975

15. Wong CC, Loewke KE, Bossert NL, et al. Non-invasive imaging of human embryos before embryonic genome activation predicts development to the blastocyst stage. Nat Biotechnol. 2010;28(10):1115-1121.

16. Athayde Wirka K, Chen AA, Conaghan J, et al. Atypical embryo phenotypes identified by time-lapse microscopy:high prevalence and association with embryo development. Fertil Steril. 2014;101(6):1637-48. 
17. Chen AA, Tan L, Suraj V, et al. Biomarkers identified with time-lapse imaging: discovery, validation, and practical application. Fertil Steril. 2013;99(4):1035-1043.

18. Kirkegaard K, Agerholm IE, Ingerslev HJ. Time-lapse monitoring as a tool for clinical embryo assessment. Hum Reprod. 2012;27(5):12771285 .

19. Meseguer M, Herrero J, Tejera A, et al. The use of morphokinetics as a predictor of embryo implantation. Hum Reprod. 2011;26(10):26582671 .

20. Cruz M, Garrido N, Herrero J, et al. Timing of cell division in human cleavage-stage embryos is linked with blastocyst formation and quality. Reprod Biomed Online. 2012;25(4):371-381.

21. Conaghan J, Chen AA, Willman SP, et al. Improving embryo selection using a computer-automated time-lapse image analysis test plus day 3 morphology:results from a prospective multicenter trial. Fertil Steril. 2013;100(2):412-419.

22. Rubio I, Kuhlmann R, Agerholm I, et al. Limited implantation success of direct-cleaved human zygotes:a time-lapse study. Fertil Steril. 2012;98(6):1458-1463.

23. Egea RR, Puchalt NG, Escriva MM, et al. OMICS:Current and future perspectives in reproductive medicine and technology. J Hum Reprod Sci. 2014;7(2):73-92.

24. Scalici E, Gala A, Ferrieres A, et al. Does embryo morphology constitute a reliable criterion for embryo selection? Gynecol Obstet Fertil. 2014;42(9):661-664.

25. Thouas GA, Dominguez F, Green MP, et al. Soluble ligands and their receptors in human embryo development and implantation. Endocr Rev. 2015;36(1):92-130
26. Cortezzi SS, Cabral EC, Trevisan MG, et al. Prediction of embryo implantation potential by mass spectrometry fingerprinting of the culture medium. Reproduction. 2013'145(5):453-462.

27. Dominguez F, Gadea B, Esteban FJ, et al. Comparative protein-profile analysis of implanted versus non-implanted human blastocysts. Hum Reprod. 2008;23(9):1993-2000.

28. Butler SA, Luttoo J, Freire MO, et al. Human chorionic gonadotropin (hCG) in the secretome of cultured embryos:hyperglycosylated hCG and hCG-free beta subunit are potential markers for infertility management and treatment. Reprod Sci. 2013;20(9):1038-1045.

29. Mains LM, Christenson L, Yang B, et al. Identification of apolipoprotein A1 in the human embryonic secretome. Fertil Steril. 2011;96(2):422427.

30. Dominguez F, Meseguer M, Aparicio-Ruiz B, et al. New strategy for diagnosing embryo implantation potential by combining proteomics and time-lapse technologies. Fertil Steril. 2015;104(4):908-914.

31. Lundin K, Ahlstrom A. Quality control and standardization of embryo morphology scoring and viability markers. Reprod Biomed Online. 2015;31(4):459-471.

32. Benkhalifa M, Madkour A, Louanjli N, et al. From global proteome profiling to single targeted molecules of follicular fluid and oocyte:contribution to embryo development and IVF outcome. Expert Rev Proteomics. 2015;12(4):407-423.

33. Hollywood K, Brison DR, Goodacre R. Metabolomics:current technologies and future trends. Proteomics. 2006;6(17):4716-4723. 